\title{
Morden Management of Inflammatory Bowel Disease in Pregnancy: A Practical Review for Obstetricians
}

\author{
Papa Essilfie \\ Hull \& East Yorkshire NHS Trust, Hull Royal Infirmary, Hull, UK \\ Email: papaessilfie@doctors.net.uk
}

Received 10 September 2014; revised 15 October 2014; accepted 26 October 2014

Copyright (C) 2015 by author and Scientific Research Publishing Inc.

This work is licensed under the Creative Commons Attribution International License (CC BY).

http://creativecommons.org/licenses/by/4.0/

(c) (i) Open Access

\begin{abstract}
Inflammatory bowel disease (IBD) is a chronic, sometimes debilitating condition that affects mainly the young population. The effect of IBD on pregnancy is therefore an important clinical issue. Many Obstetricians are likely to come across the occasional patient with this chronic condition. The following article discusses the salient factors which need to be considered in pregnant women with IBD and serves as a quick, practical but nevertheless comprehensive guide for the practicing Obstetrician.
\end{abstract}

\section{Keywords}

Crohns Disease (CD), Ulcerative Colitis (UC), Inflammatory Bowel Disease (IBD), Small for Gestational Age (SGA)

\section{Introduction}

Crohn's disease and Ulcerative colitis are two idiopathic inflammatory bowel disorders that affect young women (and men). The peak age of onset in women is between 15 and 30 years, a period of time during which many women fall pregnant. Significant anxiety is therefore understandably provoked by concerns over heritability to potential offspring, disease flares during pregnancy, adverse pregnancy outcomes and the teratogencity of some therapeutic agents. This article describes both clinical conditions (Crohn's disease and ulcerative colitis), discusses the way in which they may affect a pregnancy and reviews the literature with regard to recent developments on management in pregnancy. 


\section{Ulcerative Colitis and Crohn's Disease}

Ulcerative colitis is a relapsing non-transmural inflammatory bowel disease that is restricted to the colon. A few patients also occasionally develop illeal inflammation (backwash ileitis) which complicates differentiation from Crohn's ileocolitis. Crohn's disease is a relapsing transmural inflammatory disease of the gastrointestinal mucosa that can affect the entire gastrointestinal tract from the mouth to the anus. Typically Crohn's disease presents with discontinuous involvement of various portions of gastrointestinal tract and ultimately results in complications such as strictures, abscesses and fistulas.

\section{Atielogy \& Pathophysiology}

Inflammatory bowel disease is thought to result from an inappropriate and ongoing activation of an aberrant gastrointestinal mucosal immune system driven by normal luminal flora. This process is facilitated by a defect in the barrier function of the intestinal epithelium [1]. Although the exact cause of the disease remains elusive, it is now clear that both genetic and environmental factors play a role in the susceptibility to IBD. Many Human studies have shown an increased risk of IBD among first degree relatives of affected patients and greater concordance among monozygotic as compared to dizygotic twins [1]. The absence of simple Mendelian inheritance suggests that multiple gene products contribute to a person's risk of IBD. In addition to these genetic factors, it is now known that certain environmental factors influence the development of the disease, the most important of which are NSAIDS (which can lead to disease flares), early appendicectomy (which is associated with a reduced incidence of Ulcerative colitis) and smoking (which seems to be protective against Ulcerative Colitis but increases the risk of Crohn's disease [2] [3].

\section{Clinical Features}

IBD is a chronic condition characterised by periods of remission and relapses. Many of the symptoms of ulcerative colitis and Crohn's disease overlap. Whiles it is often possible to make a firm diagnosis of either Crohn's disease or ulcerative colitis in most cases, this distinction is occasionally impossible. The term indeterminate colitis is then applied. Ulcerative colitis usually presents with bloody diarrhoea. Crohn's disease may present with abdominal pain, fever or peri-anal disease. Occasionally it may also present with rectal bleeding and diarrhoea. A comprehensive table of the cardinal clinical features of both conditions and the frequency with which they occur is shown below (Table 1 ).

A final diagnosis of either Crohn's disease or ulcerative colitis is made based on the clinical history, physical signs, radiologic features, endoscopic features, histopathological studies and laboratory test results.

Abdominal X-ray may show fine superficial ulceration in UC. The pattern of distribution is continuous. Strictures and fistulas are rare and the ileum is usually not involved except when there is "backwash ileitis" when it may be dilated. In CD, X-ray usually shows a discontinuous or segmented pattern, ulceration is deep with submucosal extension, strictures and fistulas are common and the ileum may be narrowed or nodular.

Endoscopy may show a cobblestone appearance in CD. Aphthous and linear ulcers may be present. In UC, pseudopolyps may be present; rectal involvement is common and the mucosa often appears very friable.

Table 1. Cardinal clinical features of both conditions and the frequency.

\begin{tabular}{ccc}
\hline CLINCAL FEATURE & ULCERATIVE COLITIS & CROHN'S DISEASE \\
\hline Abdominal Pain & Sometimes & Common \\
Fever & Fairly Common & Common \\
Abdominal Mass & Never & Common \\
Peri-Anal Disease & Never & Common \\
Growth Failure & Occasional & Common \\
Signs of Malnutrition & Fairly Common & Common \\
Diarrhoea & Very Common & Fairly Common \\
Rectal Bleeding & Very Common & Fairly Common \\
\hline
\end{tabular}


Laboratory tests may show a high ESR/CRP (especially during flares). Antineutrophil cytoplasmic antibodies are common in UC and anti Saccharomyces cerevisiae antibodies are common in CD. These serologic tests are however not recommended for routine diagnosis.

\section{Pregnancy Related Issues}

\subsection{Disease Activity during Pregnancy}

One of the important factors which influence IBD disease activity during pregnancy is the state of the disease at conception or shortly before pregnancy. Pregnancy "per se" has not been shown to increase the likelihood of disease flares [4]. When conception takes place during a period of disease quiescence, the probability of a flare during pregnancy is similar to the expected risk of flare in the non-pregnant patient. A large American populationbased Cohort study of IBD patients showed a majority of patients had inactive or mild disease during pregnancy [4]. When conception occurs during active disease however, some two-thirds of patients will have active disease in pregnancy and of those patients with active disease a further two-thirds will have disease flares [5]. This emphasizes the importance of maintaining disease remission prior to pregnancy and makes the role that the primary carers of these patients (GP's and gastroenterologists) play, crucial.

Generally the overall course of the disease (particularly quiescent disease) seems to be slightly milder in pregnancy. The mechanism for the potential beneficial effect of pregnancy on the disease remains elusive. Immunosuppression induced by a disparity of some HLA (Human Leukocyte Antigen) loci between mother and fetus has been suggested as an important factor [6].

\subsection{Heritability}

Several clinical observations suggest that genetic factors play a significant role in the susceptibility to inflammatory bowel disease. Children born to parents with IBD are genetically predisposed to developing IBD themselves. When one parent is affected, the overall risk of IBD in the offspring appears to be 2 - 13 times higher than in the general population [7]. When both parents are affected the lifetime risk of a child rises to 36\% [7]. Prenatal genetic testing is currently not available. This information (of increased lifetime risk of the disease for potential children) will therefore have to be shared with the expectant mother and her partner in a sensitive fashion. Mode of delivery may influence the risk of IBD in the child. New emerging data suggest that caesarean section may increase the risk of Crohn's disease in children born to parents affected by the disease. The explanation for this increase may lie in the alteration of the normal bacterial colonization of the newborn's intestine during caesarean section.

\subsection{Perinatal Outcome}

There is no evidence that IBD increases the risk of congenital malformation. Recent data from America also suggest that the risk of spontaneous miscarriage is not increased in women with IBD. The evidence with regards to preterm delivery, low birth weight (LBW) small for gestational age (SGA) and intra-uterine growth restriction (IUGR) in quiescent disease is conflicting. Whiles some European studies have shown an increased incidence of preterm birth, LBW and SGA in IBD [7], other studies have shown no such increase [8]. Most of these studies however concur that the occurrence of disease flares during pregnancy increases the risk of adverse perinatal outcomes such as preterm delivery, LBW and SGA. The exact mechanism for this increased perinatal morbidity in the presence of disease flares is unknown. It has been hypothesized that high concentrations of prostaglandins in UC flares might stimulate preterm birth. It has also been suggested that active bowel disease might stimulate preterm birth through nutritional deficiency. The caesarean section rate is slightly increased in patients with IBD but this may reflect Obstetrician preference in the modern medicolegal climate rather than medical necessity. IBD increases the risk of venous thromboembolism in pregnancy. Appropriate thromboprohylaxis must therefore be considered if these patients are hospitalized for prolonged periods. Current data makes a firm conclusion on other maternal complications such as preeclampsia, APH, and chronic hypertension difficult to reach.

\subsection{Treatment}

Medical management remains the mainstay of treatment for patients with IBD. Surgical management may occa- 
sionally be warranted if life threatening complications (e.g. bowel perforation, refractory rectal bleeding, and toxic megacolon unresponsive to medical therapy) ensue. When there is an appropriate indication for surgery during pregnancy, the risk of on-going illness generally outweighs the periprocedural risk for the fetus. In pregnancy, a temporary ileostomy rather than primary anastomosis is often advocated. The use of Turnbull-Blowhole colostomy for colonic decompression/illeal diversion, with delayed restorative protoclectomy and IPAA (illeal Pouch Anal Anastomosis) has also been suggested for women less than 28 weeks gestation. In women close to Term gestation, synchronous caesarean section with subtotal colectomy is possible. Life threatening complications are rare in pregnancy nevertheless it is important for Obstetricians to be aware of these complications as they may be the first doctors to come into contact with these patients when such complications occur.

The principles of medical therapy are firstly to induce remission and then to maintain remission for prolonged periods of time. An expanding number of drugs are now available for targeting the inflammatory process, controlling active disease and maintaining remission.

As a general rule medical therapy for IBD should be continued during pregnancy as the benefits of stable remission largely outweigh the potential risks in most cases. There are a limited number of high quality studies with regard to drug safety in pregnancy and most of the human data available comes from large retrospective databases or case series.

Below are some of the common drugs and how their use may affect pregnancy.

\subsection{Aminosalicylates}

Mesalamine (Asacol) and Sulfasalazine are two of the common aminosalicylates used in the treatment of IBD. A met-analysis of 7 studies of aminosalicylates in pregnancy showed no statistically significant increase in Congenital anomalies, stillbirth, spontaneous abortion, preterm delivery or low birth weight with their use. Sulfasalazine has an inhibitory effect on dihydrofolate reductase and the potential folate deficiency which could occur with its use in pregnancy is countered with the administration of supplemental folic acid (1mg twice a day) before and throughout pregnancy. Although transfer to breast milk of sulfasalazine occurs, the amount transferred is negligible. Secondly, the sulfapyridine moiety of these drugs displaces only very little bilirubin from albumin compared to other sulphonamides, thus making the occurrence of Kernicterus only a remote possibility.

\subsection{Thiopurines}

The purine analogues, 6 mercaptopurine and its precursor, Azathioprine cross the placenta. Although animal studies have suggested that there may be an increased risk of teratogenicity (with an increased frequency of cleft lip and palate, skeletal and urogenital abnormalities), human studies have not confirmed this risk. The large CESAME study published in 2011 showed no increased risk of congenital abnormalities when thiopurines were used in pregnancy. The PIANO study which was a multicenter prospective study with more than 1000 IBD pregnant women also confirmed this re-assuring finding, showing no increased risk of congenital malformation. The protection that human foetuses have may be due to the fact that the immature fetal liver lacks the enzyme inosinate pyrophosphorylase which is essential for the conversion of azathioprine to its active metabolite, 6 Mercaptourine. Breast feeding whilst on thiopurines has been relatively contraindicated in the past because of the risk of myelotoxicity in the newborn. However very little amounts of the drug is excreted in breast Milk. A study by Christensen et al. demonstrated peak excretion of 6MP in the first 3 hours after drug intake. Lactating mothers are therefore advised to wait 4 hours after dosing to breastfeed. Infants with a compromised immune system are probably best not breast fed.

\subsection{Corticosteroids}

Corticosteroids are often used in the setting of disease flares when other more common drugs like aminosalicylates have not been effective in controlling active symptoms. Short acting agents like prednisone, prednisolone and methylpredisolone are more efficiently metabolized by the placenta and are therefore preferred to other steroids. The use of corticosteroids is often restricted to the acute inflammatory phase of the disease as they have not been proven to have any value in maintaining remission. As much as possible steroid use should be avoided in the first trimester due to the possible risk of orofacial clefts in foetuses exposed to these agents in-utero. Other 
concerns raised by the use of steroids in pregnancy are the development of Gestational diabetes and hypertension in pregnant women. Prolonged use of steroids can also lead to the potential occurrence of adrenal insufficiency in the infant. Corticosteroids are compatible with breast feeding as only low levels of the drug occur in breast milk. An interval of at least 4 hours between intake of more than $40 \mathrm{mg}$ of steroid and breastfeeding has been recommended to further reduce the chances of adrenal insufficiency occurring in the infant.

\subsection{Biologics (Anti Tnf- $\alpha$ Therapy)}

Although these drugs are relatively new, their use is considered compatible with pregnancy. The commonest of these drugs, Infliximab (IFX) and Adalimumab (ADA) are monoclonal immunological G1 (IgG1) antibodies used in the induction and maintenance of remission in IBD. Both drugs are actively transported through the placenta via specific fetal receptors (probably the FcRn receptor). It is believed that transplacental passage becomes even more prominent after 30 weeks gestation. The presence of these drugs in the newborn has been associated with some immunosuppression and several cases of infections have been reported when live vaccines have been administered to these infants. Two large studies which have looked at INF safety in pregnancy (The TREAT Registry and the INF safety data base) and the much larger PIANO study (Pregnancy in inflammatory bowel disease and Neonatal outcomes) which looked at Anti-TNF therapy in general, did not show any increased risk of miscarriage, congenital anomaly, preterm delivery or growth restriction with the use of these drugs. Given the importance of maintaining control over disease activity, the use of these drugs should generally be continued during pregnancy. Because of the active transplacental transport of IFX and ADA particularly during late $2^{\text {nd }}$ and $3^{\text {rd }}$ trimesters, many clinicians substitute these agents with other agents after 32 weeks gestation if this is possible. It must be remembered however that the biggest risk to the pregnancy remains disease activity with its attendant risk of preterm birth and associated sequelae. If suitable substitutes cannot be found, it would be sensible to continue with these drugs. Vaccination with life vaccines is contraindicated in the first 6 months in infants whose mothers were exposed to these drugs in pregnancy. Breast feeding is acceptable with mothers on INF as only minimal amounts of the drug are found in breast milk.

\subsection{Antibiotics}

Empirical clinical experience has led to the recognition that antibiotics are useful in the treatment of patients with Crohn's disease [9]. Metronidazole in particular can be effective in patients who have Crohn's disease with peri-anal fistulas. Although a population based case-control study of infants of women exposed to metronidazole in the first trimester of pregnancy had slightly higher rates of cleft palate, 2 meta-analysis, 2 retrospective cohort studies and a prospective controlled study showed no association with birth defects. Metronidazole is transferred to breast milk and it is recommended that breast feeding is withheld for 12 - 24 hours after ingestion.

The other antibiotic group sometimes used in the treatment of IBD is the Quinolone group of antibiotics (e.g. ciprofloxacin, norfloxacin). The evidence for the efficacy of Quinolones in the treatment of IBD is limited. This group of drugs have also been associated with potential damage to joints of immature rats, dogs and children. Human studies have not shown an increased risk of congenital malformation in infants born to mothers exposed to quinolones.

Amoxicillin/clavulanic acid (Augmentin) is a safer alternative in pregnancy. It is particularly useful in the treatment of Pouchitis. Prospective studies have not shown an increased teratogenic risk and the drug is safe to use in breast feeding

\subsection{Other Agents}

CYLCOSPORINE/TACROLIMUS-Most of the literatures on the use of cyclosporine and tacrolimus in pregnancy come from patients who have had transplant surgery. A retrospective study of maternal complications and pregnancy outcome among kidney transplant patients on tacrolimus and cyclosporine showed increased rates of perinatal complications but no congenital abnormalities. Several studies and a met analysis have also confirmed that cyclosporine is not a Human teratogen [10]. The ability of the drug to control disease activity in UC, and defer bowel resection thereby allowing pregnancy to progress to term has been demonstrated by several studies. Cyclosporine should be avoided by breastfeeding mothers. The drug is detected in breast milk and can potentially cause toxicity and immunosuppression in infants. 


\subsection{Methotrexate}

Methotrexate is a teratogen and is contraindicated in pregnancy. Effective contraception is vital when females of child-bearing age are put on methotrexate. The drug should be stopped 3 months (and preferably 6 months) before conception. When accidental pregnancy occurs in a woman on methotrexate, the drug should be stopped immediately. Termination of pregnancy is not mandatory (as normal pregnancy outcome has been reported in women exposed to methotrexate in pregnancy) but must be discussed. High dose folic acid should be prescribed for patients in whom the pregnancy is not terminated.

\subsection{Management in Pregnancy}

Most women with IBD will have a normal pregnancy and deliver a healthy infant at Term. Because of the adverse outcomes that can occur in these patients however, it is appropriate that these pregnancies are managed as High risk. IBD in pregnant women is one of the medical conditions which make multidisciplinary care mandatory. Pregnant women with IBD should be cared for by Obstetricians with special interest in medical disorders, gastroenterologists and midwives. In our Unit, these patients are seen in a setting with all these Clinicians present so that the number of hospital visits by the patients can be kept to a minimum.

\subsection{Preconception}

Many studies have shown that disease activity at conception is extremely important in determining perinatal outcome and the risk of flares in pregnancy. This underscores the importance of maintaining disease remission (or at least stable disease activity) prior to conception. Since the role of "Pre-Conception" clinics have not been well established in Hospital care in the UK, this role often falls to the patient's GP (General Practitioner) and gastroenterologist. Pregnancy in women with IBD should be a planned event. It is the responsibility of the GP to ensure that adequate contraception is maintained in women of childbearing age not planning to embark on pregnancy.

\subsection{Antenatal Care}

Booking should be as early as possible. All the usual booking blood tests (Blood count, blood group/antibody screen and infection screen) should be requested at booking. Dating scan is performed between 9 - 12 weeks. A Nuchal translucency (NT) scan is usually performed around the same time. Immunization status should be checked and be up to date. In patients with IBD, vaccination (particularly live vaccination) should be organised prior to pregnancy if the patient is not on an anti-TNF agent.

The current state of the disease and the patient's drug regimen must be reviewed at booking. Medications which are contra-indicated in pregnancy should be stopped immediately and substituted with more "pregnancy compatible" medications. Any change of medication (e.g. switching sulfasalazine to mesalamine to minimize anti-folate effects) must be discussed with the patient. Potential drug changes at a later date in the pregnancy (e.g. cessation of INF and ADA in the third trimester to limit the transplacental transfer of these agents) must also be discussed and documented in the patient's notes at booking. A plan for how Antenatal care would be managed and the times for other antenatal appointments should be documented. The opportunity must be taken to discuss intra-partum care as well as the immediate post partum care. In our unit the patients are often seen in a Medical Disorders clinic with a gastroenterologist and an Obstetrician with interest in Medical disorders present. A fetal anatomy scan is performed at about 20 weeks. If a teratogenic agent has been used up to 3 months prior to conception an initial scan may be organized at 16 weeks in addition to the usual 20 -week fetal anatomy scan. In this instance a fetal cardiac echocardiogram may also be performed at 22 weeks. If the disease is quiescent during pregnancy, the patient is usually seen monthly until 28 weeks, fortnightly until 36 weeks and then weekly until term. Fetal growth scans are unnecessary when the disease is quiescent and stable. Induction of Labour should be restricted to Obstetric indications.

Disease flares and exacerbations should be managed jointly by the gastroenterologist and the Obstetric team. Hospital admission may occasionally be warranted if symptoms are acute. Initial laboratory work up may reveal an increased CRP/ESR. The usual physiologic changes which occur in pregnancy should be borne in mind when interpreting these laboratory results.

When life threatening complications occur, radiographic studies may have to be performed (if required) in 
spite of the very small teratogenic risk posed to the fetus. The foetus is most susceptible if exposed to ionizing radiation of greater than 0.15 GY under 20 weeks gestational age. Magnetic resonance imaging (MRI) avoids fetal exposure to ionizing radiation. Current advances in MRI enterography have made it an effective, and indeed the preferred investigation in pregnancy, particularly for small bowel complications.

Endoscopic assessment may sometimes be required in pregnancy. In pregnancies over 20 weeks gestation, endoscopy should be performed with the patient in the left lateral position to avoid supine hypotension syndrome. If sedation is needed, the advice of an Obstetric anaesthetist should be sought. In the presence of significant rectal bleeding bipolar cautery (rather than monopalar cautery) may be applied as it is much safer than monopalar cautery.

Repetitive flares during pregnancy are an indication for ordering antenatal foetal growth scans as such flares may be associated with SGA foetuses.

\subsection{Delivery}

Patients with IBD have a higher rate of caesarean section compared to women in the general population [11]. There seems no medical reason why this should be so. Studies suggest that many of the caesarean sections done in IBD patients are done because of patient or Obstetrician choice. The medical indications for caesarean section in patients with IBD are

1) Active peri-anal CD;

2) Active rectal disease in a patient with UC;

3) UC patients who have undergone colectomy with IPAA (Illeal Pouch Anal Anastomosis).

In women with IPAA, vaginal delivery could potentially increase the risk of Pouch dysfunction. The mechanical forces involved in vaginal delivery could also threaten the anal sphincter and impair its function in a situation where maintenance of continence is already challenged. As much as possible, episiotomies should be avoided in patients with IBD if vaginal delivery is inevitable.

\subsection{Post Partum Care}

Post partum flares are not uncommon (particularly when women cease taking their medication because of anxiety over drug ingestion during breast feeding). In cases where disease activity has been stable and where treatment regimens have been maintained, the risk of flares in the immediate post partum period does not seem to be increased over the general rate of disease flares in IBD at any other time. Several studies have suggested that breastfeeding is safe in the setting of most IBD medications and given the well established benefits of breastfeeding in the neonate, women should generally be encouraged to breastfeed.

It is important to remember that NSAIDS (Non Steroidal Anti-Inflammatory Drugs), which are common analgesics used in the post-partum period can trigger flares and are therefore contra-indicated in patients with IBD.

The postpartum period offers a unique opportunity for Obstetrician/gynaecologists to offer advice on contraception and this opportunity must not be missed.

Advice should also be given on childhood immunization particularly if a woman has had treatment with biologic agents such as IFX and ADA during pregnancy. Life vaccines (such as Measles Mumps, Rubella varicella, smallpox and rotavirus) are contraindicated in the first 6 months of life, if an infant has been exposed to these biologic agents in utero. These agents are actively transported through the placenta especially during the $3^{\text {rd }}$ trimester and could potentially have a deleterious effect on the infant's immune response to live vaccines. Cases of fatal disseminated BCG infection after BCG vaccination have been reported.

\section{References}

[1] Podolsky, D.K. (2002) Inflammatory Bowel Disease. New England Journal of Medicine, 347, 417-428. http://dx.doi.org/10.1056/NEJMra020831

[2] Conses, J., Beaugerie, L., Carbornnel, F. and Gendre, J.P. (2001) Smoking Cessation and the Course of Crohn’s Disease: An Intervention Study. Gastroenterology, 120, 1093-1099. http://dx.doi.org/10.1053/gast.2001.23231

[3] Lindberg, E., Tysk, C., Anderson, K. and Jarnerot, G. (1988) Smoking and Inflammatory Bowel Disease: A Case Control Study. Gut, 29, 352-357. http://dx.doi.org/10.1136/gut.29.3.352

[4] Susie, W.Ng. and Mahedevan, U. (2013) Management of Inflammatory Bowel Disease in Pregnancy. Expert Rev. Clin. Immunol, 9, 161-174. 
[5] Biederman, L., Rogler, G., Varvricka, S.R., Siebold, F. and Seirafi, M. (2012) Pregnancy and Breastfeeding in Inflammatory Bowel Disease. Digestion, 86, 45-54. http://dx.doi.org/10.1159/000341941

[6] Kane, S., Kisiel, J., Shih, L., et al. (2004) HLA Disparity Determines Disease Activity through Pregnancy in Women with Inflammatory Bowel Disease. The American Journal of Gastroenterology, 99, 1523-1526. http://dx.doi.org/10.1111/j.1572-0241.2004.30472.x

[7] Kwan, L.Y. and Mahedevan, U. (2010) Inflammatory Bowel Disease and Pregnancy: An Update. Expert Review of Clinical Immunology, 6, 643-657. http://dx.doi.org/10.1586/eci.10.35

[8] Bush, M.C., Patel, S., Lapinski, R.H. and Stone, J.L. (2004) Perinatal Outcomes in Inflammatory Bowel Disease. The Journal of Maternal-Fetal and Neonatal Medicine, 15, 237-241. http://dx.doi.org/10.1080/14767050410001668662

[9] Sutherland, L., Singleton, J., Sessions, J., et al. (1991) Double Blind, Placebo Controlled Trial of Metronidazole in Crohn's Disease. Gut, 32, 1071-1075.

[10] Bar Oz, B., Hackman, R., Einarson, T., et al. (2001) Pregnancy Outcome after Cyclosporin Therapy during Pregnancy: A Metanalysis. Transplantation, 71, 1051-1055. http://dx.doi.org/10.1097/00007890-200104270-00006

[11] Ilnyckyji, A., Blanchard, J.F., Rawsthorne, P., et al. (1999) Perianal Crohn's Disease and Pregnancy: Role of Mode of Delivery. The American Journal of Gastroenterology, 94, 3274-3278. 
Scientific Research Publishing (SCIRP) is one of the largest Open Access journal publishers. It is currently publishing more than 200 open access, online, peer-reviewed journals covering a wide range of academic disciplines. SCIRP serves the worldwide academic communities and contributes to the progress and application of science with its publication.

Other selected journals from SCIRP are listed as below. Submit your manuscript to us via either submit@scirp.org or Online Submission Portal.
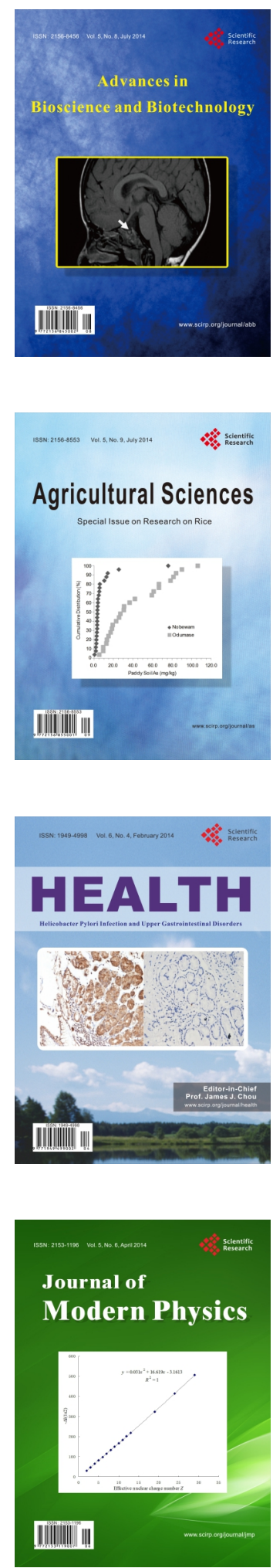
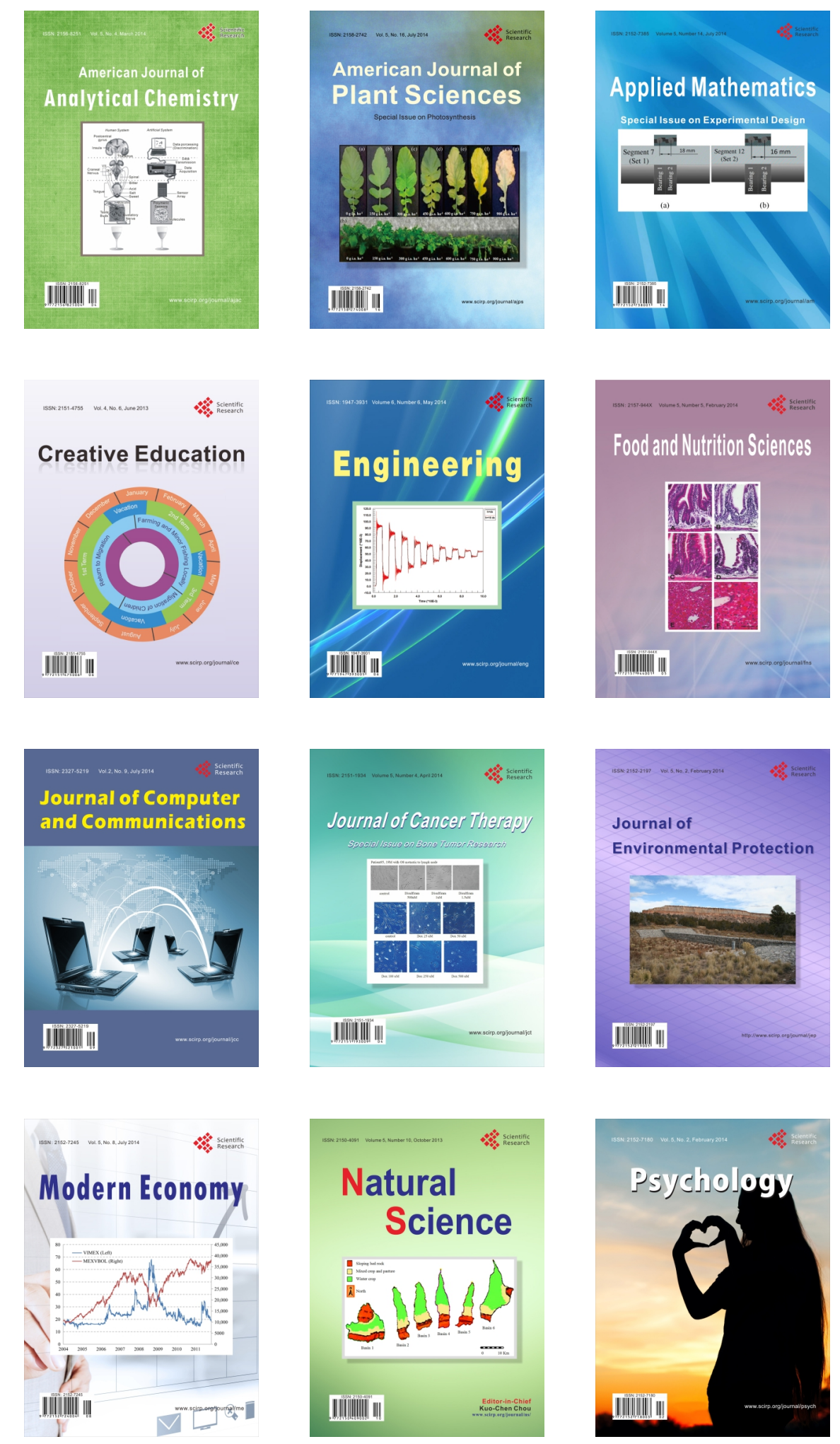